\title{
Increased expression of activating receptors and up-regulated function of natural killer cells in peripheral blood of patients with HBV-related hepatocellular carcinoma
}

\author{
Yan-tao Pei \\ Shijiazhuang Fifth Hospital \\ Wen-fang Yuan \\ Shijiazhuang Fifth Hospital \\ Qian Zhang \\ Shijiazhuang Fifth Hospital \\ Xin-yan Yue \\ Shijiazhuang Fifth Hospital \\ Xiu-li Chen (D 13363887189@163.com) \\ https://orcid.org/0000-0002-7430-7852 \\ Lin Jia \\ Hebei Hospital of Traditional Chinese Medicine
}

Research article

Keywords: hepatocellular carcinoma, natural killer cells, activating receptors, inhibitory receptors, peripheral blood

Posted Date: April 22nd, 2020

DOI: https://doi.org/10.21203/rs.3.rs-18760/v1

License: (c) (i) This work is licensed under a Creative Commons Attribution 4.0 International License. Read Full License 


\section{Abstract \\ Background}

This study aimed to investigate and identify the characteristics of peripheral natural killer (NK) cells of hepatocellular carcinoma (HCC) patients who infected with hepatitis B virus.

\section{Methods}

Flow cytometry was utilized to identify the frequency and receptors of NK cells, in the meantime, to analyze the killing ability and cytotoxicity of NK cells of patients of which embraced 36 cases of HBVassociated HCC, 34 individuals who suffered in HBV-associated cirrhosis (LC) and 30 non-liver dysfunction healthy individuals as control (HC).

\section{Results}

Cell counts for NK and CD $56^{\text {dim }}$ NK were reduced in patients with HCC, however, there was no statistically significant. The count of CD56 bright NK cells in the HCC subpopulation was remarkably higher than the subset of the HC $(P<0.05)$. The counts of activated receptors of NKG2D/p30 were elevated in patients with HCC, as well as the NKp44 and the NKp46. There was no statistically meaningful difference of inhibitory receptor expressions such as CD158a/b on peripheral NK cells of patients with HCC and LC and those with $\mathrm{HC}(P>0.05)$. Following an IL-12 stimulation, the production of INF- $\gamma /$-a of patients with HCC was less than those produced by $\mathrm{HC}(\mathrm{P}<0.05)$. However, killing activity and cytotoxicity, as the primary responsibilities of the natural killer cells, were upregulated in HCC individuals. HBV associated individuals were found lower counts and capability to produce cytokines of natural killer cells. Nevertheless, the killing capacity and cytotoxicity of NK cells were stronger than those of the HC group. This may be associated with the increased activating receptors expression of NK cells.

\section{Conclusions}

This study demonstrated that the activating receptors expression and the cytotoxicity of NK cells in the blood were independent predictors of development in $\mathrm{HCC}$ patients, and the recovery of NKG2D+CD $56^{\mathrm{dim}}$ NK cells could increase the prognostic value.

\section{Background}

Based on the date from the WHO (http://gco.iarc.fr/today/data/factsheets/cancers), liver malignancy has been the third most common death cause world widely. Hepatocellular carcinoma (HCC) contributes around 85 percent to 90 percent of the primary liver malignant tumors [1], and about 85 percent of 
attributable causes are chronic virial infection such as Hepatitis B (HBV) in China, due to the high prevalence of HBV infection [2]. To date, resection, transcatheter arterial chemoembolization, radiofrequency ablation, stereotactic radiation therapy etc. have been applied widely in the treatments for HCC. However, the outcome of HCC is still unsatisfied since its stubbornly high recurrence rate, which partially caused persistent dysfunction of the antitumor immune response, for example, the dysfunction of immunologic response [3].

Natural killer (NK) cells serve as the primary constituent of the human immunology system. NK cellmediated innate immunity is maybe crucial in hosting defense against cell malignancy transformation [4, 5]. Since the strongly cytolytic activities of liver-based NK cells have been considered to be essential for HCC immune surveillance [6, 7], functional NK cell capacity probably influences HCC recurrence and prognosis $[8,9]$. During the HCC occurrence and development, the distribution and absolute count of NK cells in the liver declined dramatically, which is significantly associated with poor outcomes of liver malignancy. Besides, the capability of NK cell recognition and killing is caused by the balance of activators and inhibitors, which undertake the sensors of pathogens and cancer cells $[10,11]$.

NK cells express varieties of receptors that transduce inhibitors either activators and identify the specific ligands on codocytes. The dormitory activating receptors, such as CD16 and NKp30/G2D/p46/44 which can recognize the ligands, such as UL-16 protein-ligand family (ULBPs), the major histocompatibility complex (MHC) category I and polypeptide-related sequence A/B to trigger cytotoxic functions and exert antitumor effects of NK cells. In parallel, NKG2A and killer immunoglobulin receptors (KIRs), such as

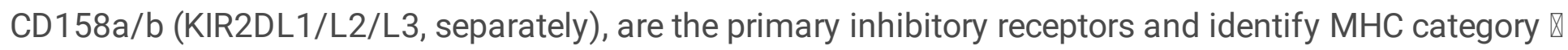
molecules in the majority. Generally, the activated NK cells are mediated by the inhibitors which combined

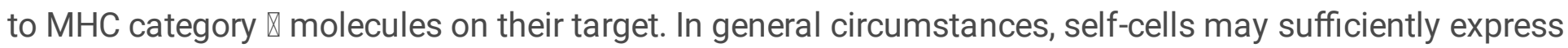

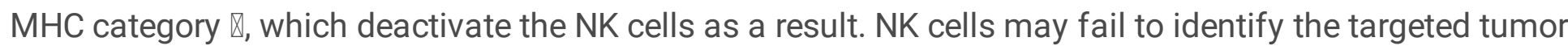
cells since they lack expression of MHC-I, which is a common process of self-recognition loss [10].

It has been known that NK cells utilize strong cytotoxicity against malignant cells, the latter can also regulate the expression of receptors either activation or inhibitors and impair the cytotoxicity function of the natural killer cells. Downregulated expression of activating receptor NKG2D can be related to declined NK cell function and miscarriage recovery of circulating NKG2D ${ }^{+}$natural killer cells in HCC after hepatectomy predicts early recurrence [8]. The significantly decreased NKp30 expression demonstrates an escaping mechanism correlated with declined NK cellular activities and cancer development [12]. In addition, these variations are not restricted to activators on NK cells. Some studies had shown that NK cell impairment was associated with upregulated receptors expression such as NKG2A, KIR2DL1/L2/L3 [13-15]. In the liver, NK cells enrich remarkably, where the importance of cytotoxicity and antitumor immunomodulation induced by those cells is broadly recognized. The alterations of the incident and phenotype features of NK cells under the circumstance of HBV-associated HCC are still unclear. This study has identified and demonstrated the different NK cell receptors' expressions and the NK cell killing ratio in HBV-associated HCC individuals, as well as how to influence the activating procedure of NK cells 
through the changes of the receptors. This may provide evidence support for the adoptive immunotherapy of HCC.

Natural killer cells can be grouped into different subpopulations according to the expression on the cellular surface markers such as CD16/56. Five NK cell subsets can be identified in human peripheral blood: CD 56 ${ }^{\text {bright }} \mathrm{CD} 16^{-}, \mathrm{CD} 56^{\text {bright }} \mathrm{CD} 16^{\mathrm{dim}}, \mathrm{CD} 56^{\text {dim }} \mathrm{CD} 16^{-}, \mathrm{CD}^{56 \mathrm{dim}} \mathrm{CD} 16^{\text {bright }}$, and CD56-CD $16^{\text {bright }}$, which were performed according on certain expression of the cellular surface markers CD16 (FcyR III, a cluster of differentiation molecule found on the surface of natural killer cells, as well as low-affinity receptor for the Fc portion of immunoglobulin G) and CD56 [16-18]. In healthy individual's peripheral blood, the CD56 ${ }^{\text {dim }}$ CD $16^{\text {bright }}$ NK cells occupy in the majority of $90 \%$ in all natural killer cells, followed by the subsets of $\mathrm{CD}_{56}{ }^{\mathrm{dim}} \mathrm{CD} 16^{-}$and $\mathrm{CD}^{-} 6^{-} \mathrm{CD} 16^{\text {bright }}[16,17,19,20]$. CD $56^{\text {dim }} \mathrm{NK}$ cells largely predominate in peripheral blood ( 90\%) and rapidly demonstrate influential cytolytic activity. However, CD 56 ${ }^{\text {bright }} \mathrm{NK}$ cells represent only $\sim 10 \%$ in peripheral blood but predominate in tissues. These cells are minority cytolytic but efficiently secrete cytokines [21]. Certain cytokines (IL-12/15/18) have been assumed to control the function of NK cells. Upon arousal with these cell factors, the cytotoxicity and the cytokine creation of NK cell subpopulations significantly rise [11]. Therefore, it is crucial to identify peripheral NK cell expression in HBV-related HCC patients.

Despite these associations, the mechanism of the association between NK cells and HBV-associated HCC is not fully revealed. We focused on the roles of the NK cells in antitumor immunologic responses and attempt to identify circulatory natural killer cells in HCC patients with HBV infection, involved with the absolute count, subpopulations, receptors. Subsequently, assess NK cells' immunologic mechanism within the prospective susceptible crowd.

\section{Methods}

\section{Study Subjects}

Thirty-six HCC patients with HBV infection, $34 \mathrm{HBV}$-associated cirrhosis (LC), and 30 healthy individuals as control (HC) were recruited consecutively from Shijiazhuang Fifth Hospital during June 2016 and December 2017 and followed up until December 2018. The HCC was diagnosed based on the its standard [22]. HBC objects conformed to the guideline of prophylaxis and treatment for chronic B-type virial hepatitis [23]. All the HCC and LC patients were positive for serum HBsAg and negative for hepatitis $\mathrm{C}$ and HIV during the recruitment. Enrolled healthy individuals undertook physical examination to exclude liver diseases and(/or) virus infestation were grouped as HC. Table 1 has listed the clinical and laboratory examinations of all the participants. Peripheral Mononuclear cells (PBMCs) were collected and isolated from all participant individuals. This study was approved by the Ethics Committees of Shijiazhuang Fifth Hospital, Prior to the study, written informed consent was obtained from individual or guardian participants. 
Table 1

Clinical and laboratory examinations of all the participants

\begin{tabular}{|c|c|c|c|c|}
\hline Variables & $\begin{array}{l}\text { HCC } \\
(n=36)\end{array}$ & $\begin{array}{l}\text { LC } \\
(n=34)\end{array}$ & $\begin{array}{l}\mathrm{HC} \\
(n=30)\end{array}$ & $P$ value \\
\hline Age (years) & $55.75 \pm 9.11$ & $47.54 \pm 15.29$ & $44.27 \pm 13.38$ & 0.0087 \\
\hline \multicolumn{5}{|l|}{ Sex, n (\%) } \\
\hline Female & $6(16.67 \%)$ & $6(17.65 \%)$ & $6(20 \%)$ & 0.948 \\
\hline Male & $30(83.33 \%)$ & $28(82.35 \%)$ & $24(80 \%)$ & \\
\hline BMI & $22.80 \pm 3.26$ & $23.41 \pm 2.89$ & $21.56 \pm 3.15$ & 0.4556 \\
\hline \multicolumn{5}{|l|}{ Blood routine } \\
\hline $\mathrm{RBC}\left(10^{12} / \mathrm{L}\right)$ & $4.12 \pm 0.47$ & $4.31 \pm 1.25$ & $5.15 \pm 1.06$ & 0.3978 \\
\hline WBC $\left(10^{9} / \mathrm{L}\right)$ & $4.35 \pm 2.86$ & $3.81 \pm 1.11$ & $7.78 \pm 1.56$ & $<0.001$ \\
\hline $\operatorname{PLT}\left(10^{9} / \mathrm{L}\right)$ & $116.55 \pm 61.93$ & $105.31 \pm 45.23$ & $198.45 \pm 54.36$ & $<0.001$ \\
\hline $\mathrm{Hb}(\mathrm{g} / \mathrm{L})$ & $131.35 \pm 15.55$ & $135.23 \pm 18.35$ & $145.35 \pm 20.48$ & 0.4589 \\
\hline HBV DNA (lg IU/mL) & $4.61 \pm 1.34$ & $5.25 \pm 1.54$ & N.A. & 0.0675 \\
\hline \multicolumn{5}{|l|}{ Liver function index } \\
\hline ALT (IU/mL) & $34(13-137)$ & $61(21-568)$ & N.A. & 0.0059 \\
\hline AST (IU/mL) & $37(18-197)$ & $75(19-467)$ & N.A. & 0.0042 \\
\hline $\operatorname{ALP}(\mathrm{IU} / \mathrm{mL})$ & $46(25-751)$ & $35(23-254)$ & N.A. & 0.2874 \\
\hline GGT (IU/mL) & $97.5(25-328)$ & $78(28-215)$ & N.A. & 0.0784 \\
\hline $\mathrm{CHE}(\mathrm{U} / \mathrm{L})$ & $4669.70 \pm 1447.01$ & $4580.12 \pm 1865.56$ & N.A. & 0.8236 \\
\hline AFP $(\mu \mathrm{g} / \mathrm{L})$ & $7.6(1.3-20000)$ & $4.5(1.5-156)$ & N.A. & $<0.001$ \\
\hline Total protein $(\mathrm{g} / \mathrm{L})$ & $68.40 \pm 6.81$ & $71.54 \pm 6.98$ & N.A. & 0.0594 \\
\hline Albumin $(\mathrm{g} / \mathrm{L})$ & $35.85 \pm 4.68$ & $36.31 \pm 6.71$ & N.A. & 0.7392 \\
\hline Globulin $(\mathrm{g} / \mathrm{L})$ & $32.55 \pm 5.47$ & $33.64 \pm 6.04$ & N.A. & 0.4310 \\
\hline TBil $(\mu \mathrm{mol} / \mathrm{L})$ & $23.62 \pm 14.70$ & $19.71 \pm 11.65$ & N.A. & 0.2234 \\
\hline
\end{tabular}

ALT: alanine aminotransferase; AST: aspartate aminotransferase; ALP: alkaline phosphatase; GGT: gamma-glutamyl transferase; AFP: alpha fetal protein; TBil: total bilirubin; DBil: direct bilirubin; PTA: prothrombin time activity; Data are median (minimum-maximum); BMl: body mass index, in kilograms, divided by height squared (in meters); RBC: red blood cells; WBC: white blood cells; PLT: platelet; $\mathrm{Hb}$ : hemoglobin; NA: not applicable. P-value refers to a comparison between HCC patients with $\mathrm{HC}$. 


\begin{tabular}{|c|c|c|c|c|}
\hline Variables & $\begin{array}{l}\text { HCC } \\
(n=36)\end{array}$ & $\begin{array}{l}\text { LC } \\
(n=34)\end{array}$ & $\begin{array}{l}\mathrm{HC} \\
(n=30)\end{array}$ & $P$ value \\
\hline $\mathrm{DBil}(\mu \mathrm{mol} / \mathrm{L})$ & $10.52 \pm 7.53$ & $8.48 \pm 5.89$ & N.A. & 0.2154 \\
\hline PTA (\%) & $82.84 \pm 13.42$ & $78.63 \pm 17.96$ & N.A. & 0.2687 \\
\hline \multicolumn{5}{|c|}{ Child-Pugh classification } \\
\hline Grade A & 24 & 19 & N.A. & 0.2480 \\
\hline Grade B & 12 & 15 & N.A. & \\
\hline \multicolumn{5}{|c|}{$\begin{array}{l}\text { ALT: alanine aminotransferase; AST: aspartate aminotransferase; ALP: alkaline phosphatase; GGT: } \\
\text { gamma-glutamyl transferase; AFP: alpha fetal protein; TBil: total bilirubin; DBil: direct bilirubin; PTA: } \\
\text { prothrombin time activity; Data are median (minimum-maximum); BMI: body mass index, in } \\
\text { kilograms, divided by height squared (in meters); RBC: red blood cells; WBC: white blood cells; PLT: } \\
\text { platelet; Hb: hemoglobin; NA: not applicable. P-value refers to a comparison between HCC patients } \\
\text { with HC. }\end{array}$} \\
\hline
\end{tabular}

\section{PBMC Isolation And Cell Purification}

Mononuclear cells were collected and then isolated by applying the Ficoll-Hypaque centrifugation (Sigma, USA) based on the manufacturer's instruction and stored in liquid nitrogen. Autologous NK cells were thereafter isolated and purified by application of the MACS Natural Killer cell negative selection kit (MACS, Miltenyi Biotec, China), according to the manufacturer's instruction. Flow cytometry was utilized which measured and confirmed the purity of NK cells $\left(\mathrm{CD}^{-}{ }^{-} \mathrm{CD}_{56}{ }^{+}\right)$was higher than $95 \%$.

\section{Frequency And Number Of NK Cell Detection}

$20 \mathrm{~mL}$ fasting venous blood was collected in a heparin anticoagulation tube from each person. The ratio and count of NK cells were detected by the IMK MultiTEST kit (BD Biosciences, USA) and TruCOUNT absolute counter tube and analyzed using FACS MULTISET software (BD Biosciences, USA), in conformity with the manufacturer's instructions.

\section{Flow Cytometry Analysis Of NK Cell Receptors}

$3 \mathrm{~mL}$ PBMCs were prepared to detect NK cell receptors. Flow cytometry was introduced to make detection and measurement. The PBMCs were thawed initially. They were resuspended in complete RPMI1640 medium (Corning Cellgro, USA) which embraced with $10 \%$ fetal bovine serum, $1 \%$ glutamine and $1 \%$ penicillin and streptomycin. $1 \times 10^{6}$ cells were seeded for every panel and treated in the dark to stain at room temperature $\left(20 \sim 25^{\circ} \mathrm{C}\right)$ for thirty minutes. NK cell phenotype identification of NK cell was performed via certain monoclonal antibodies which were: FITC conjugated anti-CD158b, PerCP-Cy5.5 
conjugated anti-CD56, PB conjugated anti-CD3, BV510 conjugated anti-CD45, APC-Cy7 conjugated antiCD16, APC conjugated anti-NKG2D/CD158a/NKP44/NKP30 and PE-Cy7 conjugated anti-NKP46. All antibodies were acquired from BD Biosciences (San Jose, CA, USA). The PBMCs have washed twice with PBS fowling staining. A BD LSR II Fortessa flow cytometer (BD Biosciences, San Jose, CA, USA) was utilized for detection and identification. The data were consequently examined via the software FlowJo (TreeStar, Ashland, OR, USA).

\section{Degranulation Of NK Cells And IFN-ץ Detection}

The cytotoxic potentials of NK cells were assessed by CD107a degranulation. PMA ( $100 \mathrm{ng} / \mathrm{mL}$, SigmaAldrich, USA) and lonomycin ( $1 \mu \mathrm{g} / \mathrm{mL}$, Santa Cruz Biotechnology, USA) were administrated at $37^{\circ} \mathrm{C}$ for $1 \mathrm{~h}$ to stimulate the freshly isolated PBMCs $\left(5 \times 10^{5}\right.$ cells $\left./ \mathrm{mL}\right)$. Controls were set with non-stimulated PBMCs. Antibody (APC conjugated anti-CD107a) was administrated to the medium first. GolgiStop was added one hour afterward. Those cells were collected and stained after $5 \mathrm{~h}$ of incubation, together with antibodies and cytokine such as PE-conjugated anti-IFN- $y$ and FITC conjugated anti-TNF-a intracellularly. The detection and measurement were performed by flow cytometry.

\section{Cytolytic Killing Assay}

K562 cells were firstly tagged with Carboxy fluorescein succinimidyl ester (CFSE, Molecular Probes, Eugene, OR) which is a fluorescent cell staining dye. PBMCs were subsequently incubated with the CFSEtagged assay cells for six hours with at ratios of 3:1, 10:1 and 30:1 separately, where the production, as well as the target cells, were set as controls. Those cells were thereafter treated with $1 \mu \mathrm{g} / \mathrm{mL}$ of 7 aminoactinomycin D (BD Biosciences, San Jose, CA, USA) for the verification of apoptosis or necrosis.

\section{Cytokine Stimulation}

PBMCs $\left(2 \times 10^{6}\right.$ cells $\left./ \mathrm{mL}\right)$ were cultured along and together with $5 \mathrm{ng} / \mathrm{mL}$ of IL-12 for $48 \mathrm{~h}$, separately. Afterward, samples were either stained with antibodies that contrapose to activation markers or further degranulate via IFN- $y$ and TNF-a to six hours, which release assays in the expression of IL-12.

\section{Statistical analysis}

The statistic works were performed by using a statistical software Stata 12.0 (StataCorp LP, Texas, USA). The normal distribution of quantitative data was analyzed by Sktest. The t-test was elected in two-group comparisons of normal distribution data, and the results were expressed as mean \pm SEM. Rank sum test was placed for data of two-group comparisons with abnormal distribution, and the results were demonstrated as a median and inter-quartile range. Spearman test was adopted for the rank correlation 
in two-group comparisons. A chi-square test was introduced to analyze qualitative data. Statistically significant is defined when two-tailed $P$ values $<0.05$.

\section{Results}

\section{Low frequency of circulating CD56 dim NK cells and high frequency of circulating CD56 ${ }^{\text {bright }}$ NK cells in HCC cases}

All the results were shown as median (P25, P75), \%. The median of total peripheral NK cells was dwindled in HCC group $18.7(5.5,77.3)$ and LC $16.1(5.8,41.2)$ than in HC $20.5(6.7,46.1)$. Nevertheless, the variation between those three subgroups did not demonstrate a statistically significant $(P=0.121$, Fig. 1$)$. Consequently, we compared the subpopulations of NK cells between all three subgroups (Fig. 1). It revealed that the median count of CD56 ${ }^{\mathrm{dim}}$ cells was dramatically lower than those in LC subset (12.4 $(4.3,34.2))$ as compared to $\mathrm{HC}(16.5(5.6,41.8), \mathrm{P}=0.024)$. No meaningful statistical difference of CD56 dim natural kill cells between HCC and LC subpopulations $(P=0.689)$ has been observed. The median count of CD56 bright cells in HCC and LC group was significantly higher than $\mathrm{HC}(\mathrm{P}=0.006, \mathrm{P}=$ 0.017 , respectively) individuals. The difference in CD $56^{\text {bright }}$ cells between HCC and LC subgroups did not demonstrate a statistical significance $(P=0.768)$.

\section{Increased activating receptors expression and declined inhibitory receptors expression in peripheral NK cells of HCC individuals}

The activating receptors (NKG2D/p30/p44/p46) expressions and inhibitory receptors (CD158a/b) over all three subgroups were listed in Fig. 2. The inhibitory receptors of CD158a/b were downregulated in HCC conditions, compared to those in $\mathrm{HC}$ individuals, which did not demonstrate any statistically significant $(\mathrm{P}=0.495, \mathrm{P}=0.633$ and $\mathrm{P}=0.399, \mathrm{P}=0.154$ respectively). The activating receptors expression (NKG2D/p30/p44,/p46) were upregulated on peripheral natural killer cells in subpopulation with HCC (1.1, $\mathrm{P}=0.0008 ; 41.2, \mathrm{P}=0.052 ; 0.2, \mathrm{P}=0.056 ; 6.5, \mathrm{P}=0.003$; respectively), compared with $\mathrm{HC}$ subset $(0.4 ; 32.6$; $0.1 ; 2.4)$, whereas the expression of NKG2D and NKp30 were upregulated in HCC individuals, compared to condition of LC $(P=0.021, P<0.001$ respectively). However, significant increase of NKp44 $(P=0.005$ and NKP46 $P=0.0007$ ) between individuals with LC and HC (Fig. 2) was observed.

\section{Unchanged CD107a degranulation and decreased production of IFN- $y$ and TNF- $a$ in peripheral NK cells of HCC group}

To assess the cytotoxicity of peripheral natural killer cells, we detected their CD107a degranulation and secretion of IFN- $y$ and TNF-a. The results did not demonstrate a statistical difference of CD107a expression among all the enrolled groups $(P=0.545$, HCC vs $L C ; P=0.130$, LC vs HC; $P=0.341, H C C$ vs HC) (Fig. 3).

IL-12 was applied to stimulate natural killer cells in all the participant groups. The expression of CD56 dim IFN- $y$ and CD56 bright IFN- $y$ in HCC patients dramatically decreased compared to LC patients and $\mathrm{HC}(\mathrm{P}<$ 
$0.001, \mathrm{P}<0.001, \mathrm{P}<0.001, \mathrm{P}<0.001$ respectively). However, there was no meaningful statistical difference in the expression of CD56 dim IFN-y in LC and HC groups $(P=0.122)$. CD56 dim TNF-a in LC individuals was significantly decreased compared to HC subgroup $(P=0.0002)$, while there was no statistical difference between HCC and LC individuals $(P=0.113)$. The level of CD56 ${ }^{\text {bright }}$ TNF-a in patients with HCC was significantly declined compared to those in patients with $L C(P=0.030)$.

Nevertheless, no remarkable difference in the LC and HC groups $(P=0.406)($ Table 2$)$ has been observed.

Table 2

Frequency of IFN- $\gamma$ and TNF- $a$ of CD $56^{\text {bright }}$ NK and CD56 dim NK cells under IL-12 stimulus [median (P25, P75), \%]

\begin{tabular}{|lllll|}
\hline & CD56 dim IFN-y & CD56 $^{\text {bright }}$ IFN-y & CD56 dim TNF-a & CD56 bright TNF-a \\
\hline HCC & $2.0(0.2,32.3)$ & $0.2(0,1.9)$ & $17.2(0.2,69.4)$ & $0.4(0,6.4)$ \\
\hline LC & $7.4(4.4,31.2)$ & $0.4(0.1,3.6)$ & $26.9(2.3,48.2)$ & $1.3(0.1,3.5)$ \\
\hline HC & $18.8(1.2,54.3)$ & $1.0(0.1,7.7)$ & $42.2(8.8,82.5)$ & $1.0(0.1,7.6)$ \\
\hline HCC vs LC & $<0.001$ & $<0.001$ & 0.113 & 0.030 \\
\hline LC vs HC & 0.122 & 0.002 & 0.0002 & 0.406 \\
\hline HCC vs HC & $<0.001$ & $<0.001$ & $<0.001$ & $<0.001$ \\
\hline
\end{tabular}

\section{Enhanced Cytolytic Activity Of Nk Cells In Hcc Subpopulation}

We revealed the natural killer cells from HCC individuals evoked higher K562 lysis level than those of LC subgroup $(P=0.0001, P=0.0009, P=0.0009$ respectively) at the effect: target $(E: T)$ ratio of 3:1, 10:1 and 30:1, separately. While the natural killer cells from LC individuals evoked lower K562 lysis level compared to those from $\mathrm{HC}$ individuals $(P=0.019, P=0.012, P=0.012$ respectively $)$ at the $3: 1,10: 1$ and $30: 1 \mathrm{E}: \mathrm{T}$ ratio. With the increase of the E:T ratio, the cytolytic activity of natural killer cells enhanced in HCC patients gradually. The result did not express a statistical significant between NK cells in HCC and HC subgroup ( $P=0.367, P=0.279$ respectively) at the $E: T$ ratio of $3: 1$ and 10:1. However, it was observed dramatically increased cytolytic activity of NK cells at the ratio 30:1 $(P=0.016)$ (Fig. 4).

\section{Discussion}

A mountain of previous evidence, particularly from pre-clinical studies of in vitro and in vivo animal models, suggests that NK cells play functional roles in antitumor defenses. Because they have direct cytotoxic effects against malignant cells and the capability to produce cell factors that activate downstream adaptive responses effectively. Natural killer cells are the first immunologic responders against viral affection. However, a sub-normality of NK cell function may even play an unexpected role in cancer development and promotion. On the basis of the CD56 antigen density, NK cells have been divided into two subsets of CD56-dim and bright. The majority of human natural killer cells (about 90\%) are the 
dominant expression of CD16 and potent cytolytic activity of CD $56^{\text {dim }}$ cells, whereas CD $56^{\text {bright }}$ cells that produce a large body of cytokines are about $10 \%$. NK cell receptors mainly include activating receptors NKp30/p44/p46/G2D and KIR2DS, whereas inhibitors as CD158a/b, etc. The change of NK cell receptors is closely related to the cytotoxicity of NK cells, and the development and occurrence of tumors [24]. Downregulated activating receptors expression, as well as upregulated inhibitors expression in peripheral circulation and malignant tissues of HCC patients has been proved by previous investigations [25-27]. However, our study revealed that the activating receptors (NKG2D/p30/p44/p46) expressions were upregulated on NK cells of HCC patients, as compared to those in HC. The results were inconsistent with the above reports. This may be because previous studies generally examined the expression of total NK cell-activating receptors while we detected activating receptors of CD56-dim and bright expressions, respectively [28]. Moreover, a small minority of patients with HCC were treated with TACE or radiotherapy in our study, while TACE and radiotherapy could improve the activating receptors expression $[29,30]$.

Recent evidence indicates that cytokines indeed promote human NK cell activation. In this study, we used IL-12 as a stimulator to stimulate NK cells to secrete IFN- $\gamma /-a$, which can reflect the function of NK cell secreting cytokines. We found that the expression of IFN- $\gamma$ and TNF- $a$ of CD $56^{\text {bright }}$ NK cells and IFN- $\gamma$ of CD56 dim natural killer cells in the HCC sub-population was remarkably decreased than the LC subgroup, consequently, the secreting cytokines function of NK cells was impaired in HCC group [31]. The negative regulation of IFN- $\gamma$ recognizing human NK cells can inhibit the activating receptor NKG2D expression and enhance the inhibitory receptor NKG2A/B and KIR2DLI expression [32]. Our results demonstrated that the expression of NKG2D/p30 of CD56 dim peripheral NK cells of HCC individuals was higher than that in the LC group. This may be linked to the lower IFN-y expression in HCC group compared with the LC group. In HCC patients, the secretion of IFN-y decreased by NK cells, which can reduce the inhibiting effect of NKG2D, consequently the expression of NKG2D receptors was elevated.

NK cell features mainly involve antibody-dependent cellular-mediated cytotoxicity, cytolytic granulemediated cell apoptosis, and cytokine-mediated NK and CTL activation, et al. Our results displayed that the features of NK cells secreted IFN- $\gamma$ and TNF-a were damaged in peripheral blood. There was no meaningful statistical difference in CD107a between HCC and LC subpopulations, but the expression of NKG2D and NKp30 significantly increased, while the cytotoxicity of K562 cells by NK cells was strengthened. NK cells mainly kill the target via releasing the granzyme and perforin. The E: T ratio of NK cells and killing K562 cells can well reflect its killing capability. NK cells evoke apoptosis of target cells primarily through exocytosis and death receptors. CD $56^{\text {dim }}$ NK cells can release perforin to kill the target cells. Our results did not demonstrate a significant statistical in the proportion of CD56 dim natural killer cells and degranulated expression of CD107a between the HCC and LC subsets. However, activating receptors expression (NKG2D/p30) in HCC individuals was higher than that in LC subsets. The expression of NKp30 of CD56 dim NK cells was rank linked to the cytotoxicity of NK cells at the E:T ratio of 1:10. The characteristics of NK cells killing K562 cells in HCC patients involved in the high activating receptors expression on the CD56 dim NK cell surface. The expression of NK cell receptors is closely related to NK cell function. The results showed that NKG2D and NK cell receptors often had a synergistic effect when 
NK cells exerted cytotoxic effects. NKG2D can have an antitumor effect when the NK cell receptors were low or no expression. The change of NK cell receptors is highly likely related to the cytotoxicity and the development of malignancies [24].

\section{Conclusions}

The present study revealed and identified that the activating receptors expression and the cytotoxicity of natural killer cells were increased in peripheral circulation of HCC individuals, which suggested that its receptors may play critical roles in natural killer cell-induced antitumor immunity. The recovery of NKG2D ${ }^{+}$CD 56 ${ }^{\text {dim }}$ natural killer cells was linked with the outcome of HCC patients. Our results were well acquainted with the mechanisms of those receptors and HCC development. The frequency of the NKG2D ${ }^{+}$CD56 ${ }^{\text {dim }}$ NK subpopulation is highly likely a substantial biomarker for predicting the outcome of individual with HCC who was administrated a hepatectomy. This can provide theoretical support for the selection of appropriate combination immunotherapy by further regulating the cell receptor expression of NK cells.

\section{Abbreviations}

NK: natural killer cell; HCC: hepatocellular carcinoma; HBV: hepatitis B virus; ULBPs: UL-16 protein-ligand family; MHC: major histocompatibility complex; KIR: killer immunoglobulin receptor; HC: healthy controls; LC: liver cirrhosis; PBMC: peripheral mononuclear cells.

\section{Declarations}

\section{Ethics approval and consent to participate}

This study was approved by the ethics committee of Shijiazhuang Fifth Hospital. Written informed consent was obtained from individual or guardian participants.

\section{Consent for publication}

Not applicable.

\section{Availability of data and materials}

The data used to support the findings of this study are included within the article.

\section{Competing interests}

The authors declare that they have no conflicts of interest.

\section{Funding}


This work was supported by grants from the Key Medical Science Research Foundation of Hebei Province (No. 20181079) for CXL. The funder had no role in the study design, data collection and analysis, decision to publish, or preparation of the manuscript.

\section{Authors' contributions}

Study concept and design: CXL and JL; Collection and assembly of data: PYT; Performed the experiment: YWF, ZQ and YXY; Data analysis and interpretation: PYT; Manuscript writing and review: PYT, CXL and $\mathrm{JL}$. All authors have read and approved the manuscript in its current state.

\section{Acknowledgments}

We thank all patients and volunteers who participated in this study and all staff in Shijiazhuang Fifth Hospital for their help in collection of samples.

\section{References}

1. Gao Q, Zhu H, Dong L, Shi W, Chen R, Song Z, Huang C, Li J, Dong X, Zhou Y, et al. Integrated Proteogenomic Characterization of HBV-Related Hepatocellular Carcinoma. Cell. 2019;179(5):1240.

2. Prevention of Infection Related Cancer (PIRCA) Group SCoCPaC, Chinese Preventive Medicine Association; Non-communicable \& Chronic Disease Control and Prevention Society, Chinese Preventive Medicine Association; Health Communication Society, Chinese Preventive Medicine Association: Strategies of primary prevention of liver cancer in China: expert consensus. (2018). Zhonghua Yu Fang Yi Xue Za Zhi 2019, 53(1):36-44.

3. Llovet JM, Burroughs A, Bruix J. Hepatocellular carcinoma. Lancet. 2003;362(9399):1907-17.

4. Chew V, Tow C, Teo M, Wong HL, Chan J, Gehring A, Loh M, Bolze A, Quek R, Lee VK, et al. Inflammatory tumour microenvironment is associated with superior survival in hepatocellular carcinoma patients. Journal of hepatology. 2010;52(3):370-9.

5. Sui Q, Zhang J, Sun X, Zhang C, Han Q, Tian Z. NK cells are the crucial antitumor mediators when STAT3-mediated immunosuppression is blocked in hepatocellular carcinoma. Journal of immunology. 2014;193(4):2016-23.

6. Ishiyama K, Ohdan H, Ohira M, Mitsuta H, Arihiro K, Asahara T. Difference in cytotoxicity against hepatocellular carcinoma between liver and periphery natural killer cells in humans. Hepatology. 2006;43(2):362-72.

7. Tian Z, Chen Y, Gao B. Natural killer cells in liver disease. Hepatology. 2013;57(4):1654-62.

8. Gao J, Duan Z, Zhang L, Huang X, Long L, Tu J, Liang H, Zhang Y, Shen T, Lu F. Failure recovery of circulating NKG2D + CD56dimNK cells in HBV-associated hepatocellular carcinoma after hepatectomy predicts early recurrence. Oncoimmunology. 2016;5(1):e1048061.

9. Tanimine N, Tanaka Y, Kobayashi T, Tashiro H, Miki D, Imamura M, Aikata H, Tanaka J, Chayama K, Ohdan H. Quantitative effect of natural killer-cell licensing on hepatocellular carcinoma recurrence 
after curative hepatectomy. Cancer immunology research. 2014;2(12):1142-7.

10. Sun C, Sun H, Zhang C, Tian Z. NK cell receptor imbalance and NK cell dysfunction in HBV infection and hepatocellular carcinoma. Cell Mol Immunol. 2015;12(3):292-302.

11. Vivier E, Tomasello E, Baratin M, Walzer T, Ugolini S. Functions of natural killer cells. Nature immunology. 2008;9(5):503-10.

12. Fiegler N, Textor S, Arnold A, Rolle A, Oehme I, Breuhahn K, Moldenhauer G, Witzens-Harig M, Cerwenka A. Downregulation of the activating NKp30 ligand B7-H6 by HDAC inhibitors impairs tumor cell recognition by NK cells. Blood. 2013;122(5):684-93.

13. Beksac K, Beksac M, Dalva K, Karaagaoglu E, Tirnaksiz MB. Impact of "Killer Immunoglobulin-Like Receptor /Ligand" Genotypes on Outcome following Surgery among Patients with Colorectal Cancer: Activating KIRs Are Associated with Long-Term Disease Free Survival. PloS one. 2015;10(7):e0132526.

14. Martinez-Sanchez MV, Periago A, Legaz I, Gimeno L, Mrowiec A, Montes-Barqueros NR, Campillo JA, Bolarin JM, Bernardo MV, Lopez-Alvarez MR, et al. Overexpression of KIR inhibitory ligands (HLA-I) determines that immunosurveillance of myeloma depends on diverse and strong NK cell licensing. Oncoimmunology. 2016;5(4):e1093721.

15. McWilliams EM, Mele JM, Cheney C, Timmerman EA, Fiazuddin F, Strattan EJ, Mo X, Byrd JC, Muthusamy N, Awan FT. Therapeutic CD94/NKG2A blockade improves natural killer cell dysfunction in chronic lymphocytic leukemia. Oncoimmunology. 2016;5(10):e1226720.

16. Caligiuri MA. Human natural killer cells. Blood. 2008;112(3):461-9.

17. Cooper MA, Fehniger TA, Caligiuri MA. The biology of human natural killer-cell subsets. Trends Immunol. 2001;22(11):633-40.

18. Mavilio D, Lombardo G, Benjamin J, Kim D, Follman D, Marcenaro E, O'Shea MA, Kinter A, Kovacs C, Moretta A, et al. Characterization of CD56-/CD16 + natural killer (NK) cells: a highly dysfunctional NK subset expanded in HIV-infected viremic individuals. Proc Natl Acad Sci USA. 2005;102(8):2886-91.

19. Alter G, Jost S, Rihn S, Reyor LL, Nolan BE, Ghebremichael M, Bosch R, Altfeld M, Lauer GM. Reduced frequencies of NKp30 + NKp46+, CD161+, and NKG2D + NK cells in acute HCV infection may predict viral clearance. Journal of hepatology. 2011;55(2):278-88.

20. Poli A, Michel T, Theresine M, Andres E, Hentges F, Zimmer J. CD56bright natural killer (NK) cells: an important NK cell subset. Immunology. 2009;126(4):458-65.

21. Sivori S, Vacca P, Del Zotto G, Munari E, Mingari MC, Moretta L. Human NK cells: surface receptors, inhibitory checkpoints, and translational applications. Cell Mol Immunol. 2019;16(5):430-41.

22. Zhou J, Sun HC, Wang Z, Cong WM, Wang JH, Zeng MS, Yang JM, Bie P, Liu LX, Wen TF, et al. Guidelines for Diagnosis and Treatment of Primary Liver Cancer in China (2017 Edition). Liver cancer. 2018;7(3):235-60.

23. Chinese Society of Hepatology CMA, Chinese Society of Infectious Diseases CMA. The guideline of prevention and treatment for chronic hepatitis B: a 2015 update. Zhonghua Gan Zang Bing Za Zhi. 2015;23(12):888-905. 
24. Domaica Cl, Fuertes MB, Rossi LE, Girart MV, Avila DE, Rabinovich GA, Zwirner NW. Tumourexperienced T cells promote NK cell activity through trogocytosis of NKG2D and NKp46 ligands. EMBO Rep. 2009;10(8):908-15.

25. Kamimura H, Yamagiwa S, Tsuchiya A, Takamura M, Matsuda Y, Ohkoshi S, Inoue M, Wakai T, Shirai Y, Nomoto M, et al. Reduced NKG2D ligand expression in hepatocellular carcinoma correlates with early recurrence. Journal of hepatology. 2012;56(2):381-8.

26. Kohga K, Takehara T, Tatsumi T, Ohkawa K, Miyagi T, Hiramatsu N, Kanto T, Kasugai T, Katayama K, Kato $\mathrm{M}$, et al. Serum levels of soluble major histocompatibility complex (MHC) class I-related chain A in patients with chronic liver diseases and changes during transcatheter arterial embolization for hepatocellular carcinoma. Cancer Sci. 2008;99(8):1643-9.

27. Sha WH, Zeng XH, Min L. The correlation between NK cell and liver function in patients with primary hepatocellular carcinoma. Gut Liver. 2014;8(3):298-305.

28. Yonekura K, Ichida T, Sato K, Yamagiwa S, Uchida M, Sugahara S, Ito S, Abo T, Asakura H. Liverinfiltrating CD56 positive T lymphocytes in hepatitis C virus infection. Liver. 2000;20(5):357-65.

29. Demaria S, Pilones KA, Vanpouille-Box C, Golden EB, Formenti SC. The optimal partnership of radiation and immunotherapy: from preclinical studies to clinical translation. Radiation research. 2014;182(2):170-81.

30. Huang M, Wang X, Bin H. Effect of Transcatheter Arterial Chemoembolization Combined with ArgonHelium Cryosurgery System on the Changes of NK Cells and T Cell Subsets in Peripheral Blood of Hepatocellular Carcinoma Patients. Cell Biochem Biophys. 2015;73(3):787-92.

31. Wu Y, Kuang DM, Pan WD, Wan YL, Lao XM, Wang D, Li XF, Zheng L. Monocyte/macrophage-elicited natural killer cell dysfunction in hepatocellular carcinoma is mediated by CD48/2B4 interactions. Hepatology. 2013;57(3):1107-16.

32. Zhang C, Niu J, Zhang J, Wang Y, Zhou Z, Zhang J, Tian Z. Opposing effects of interferon-alpha and interferon-gamma on the expression of major histocompatibility complex class I chain-related $A$ in tumors. Cancer Sci. 2008;99(6):1279-86.

\section{Figures}


A

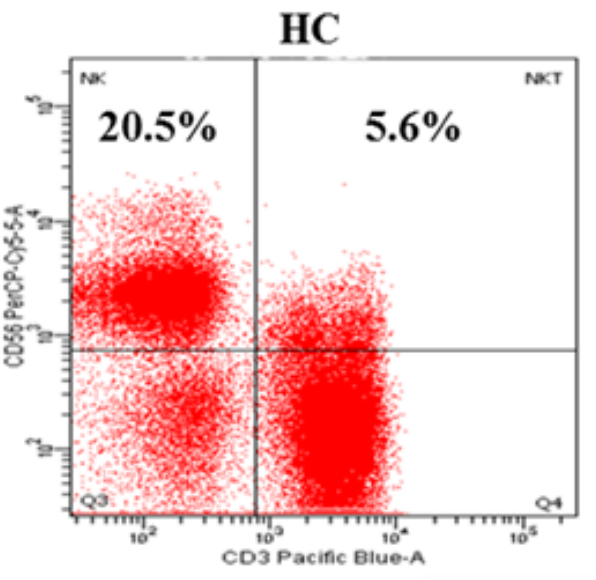

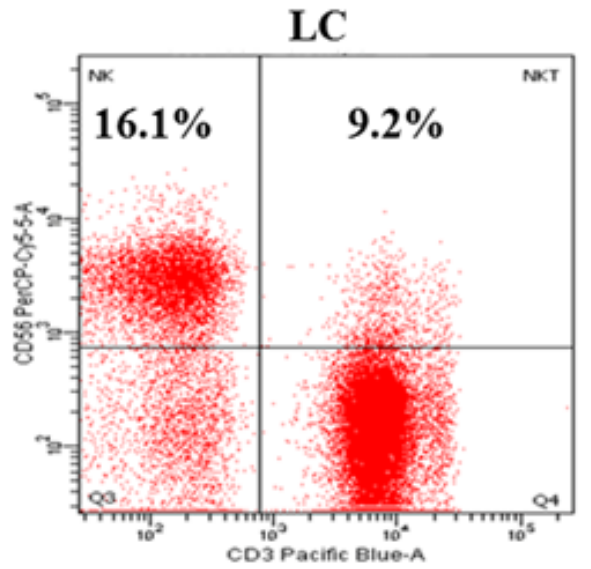

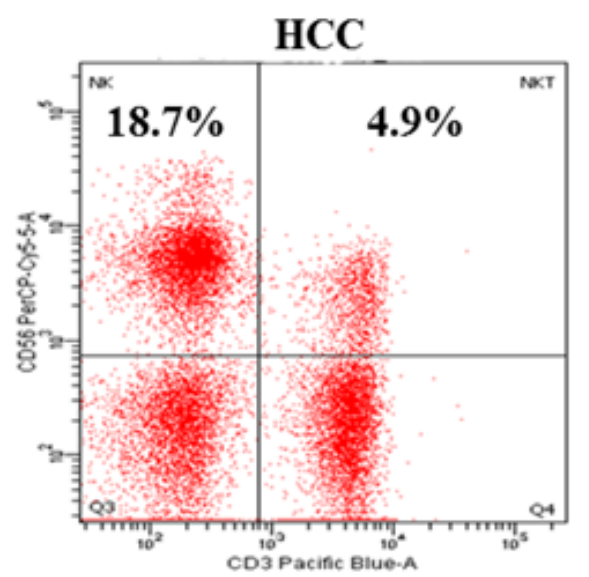

B

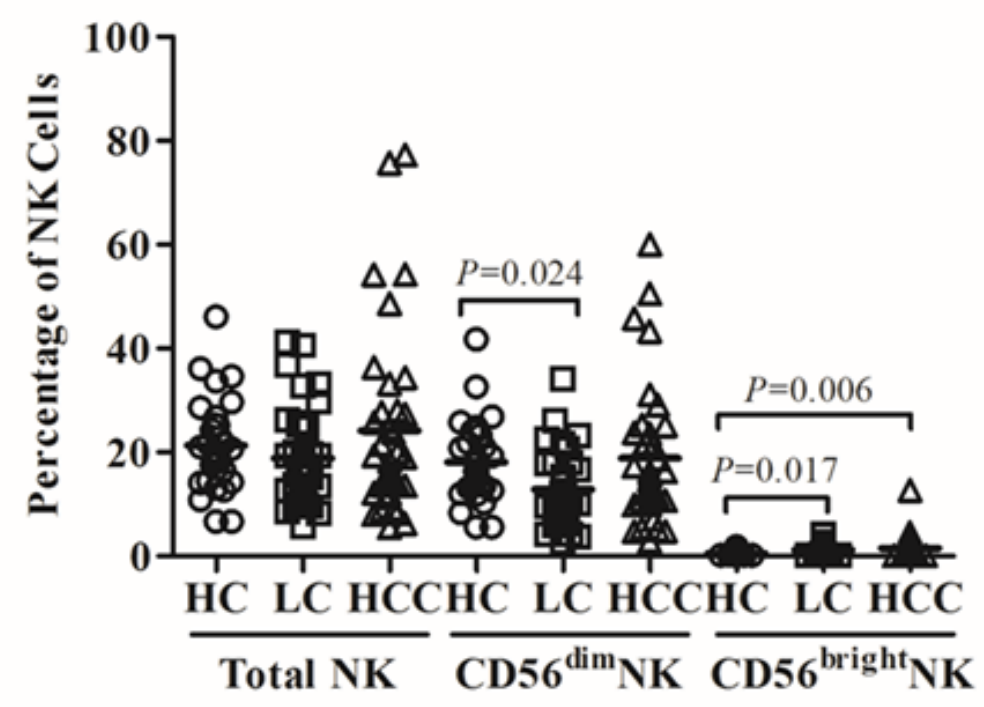

Figure 1

Peripheral NK cells stained in HCC subgroup. (A) demonstrate dot plots of natural killer cell subgroups from HC, LC and HCC individuals according to the certain expression of CD56. (B) Frequencies of natural killer cells contribute lymphocytes of three groups. HC: healthy controls; LC: Liver cirrhosis; liver cancer: HCC. 
A

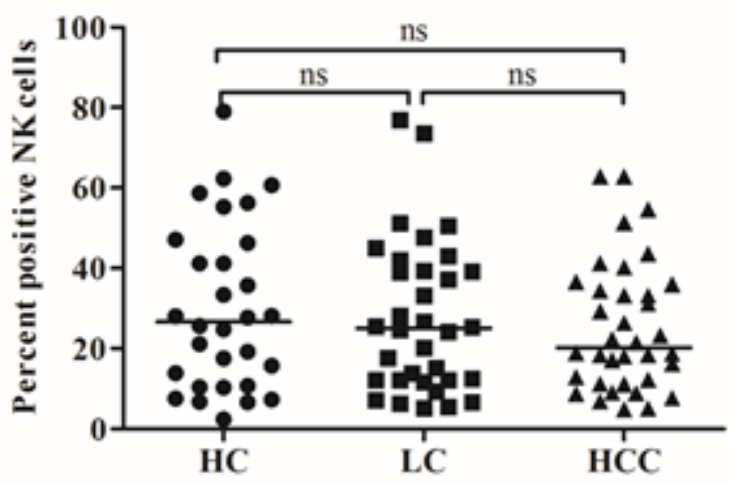

B

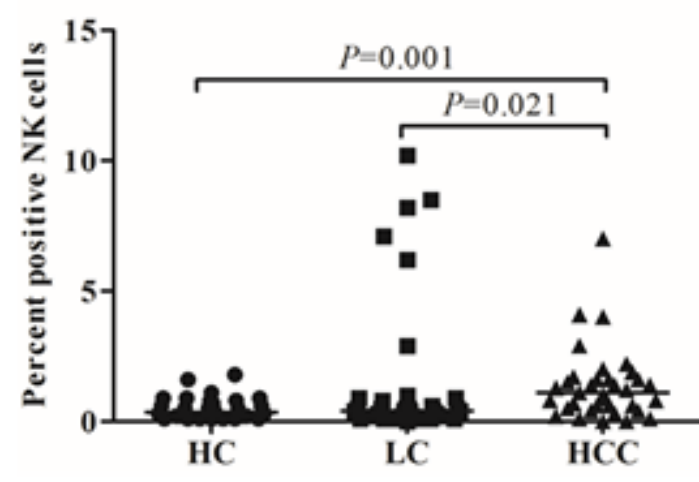

NKp44

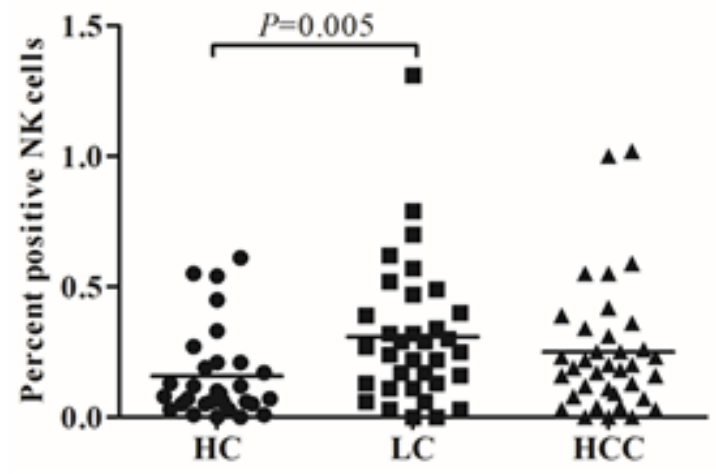

CD158b

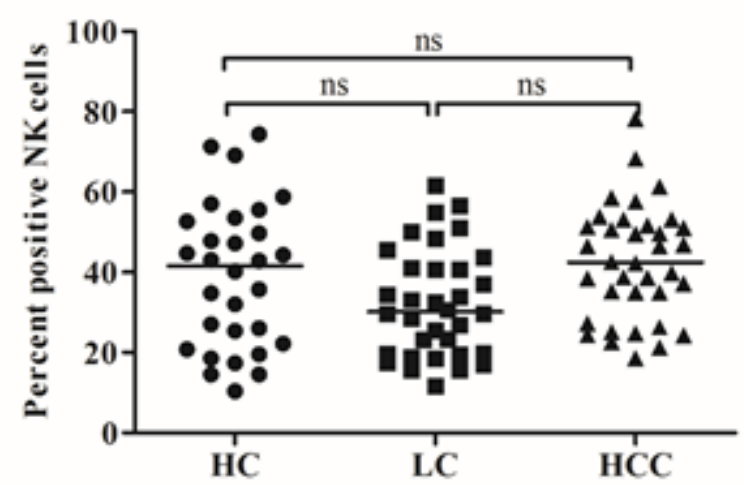

NKp30

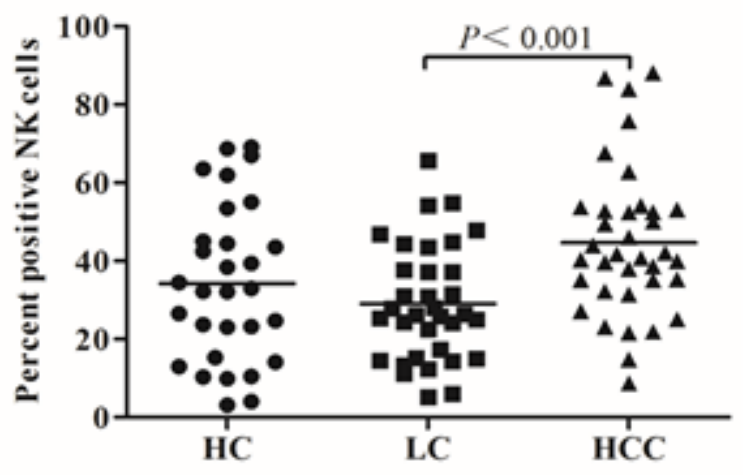

NKp46

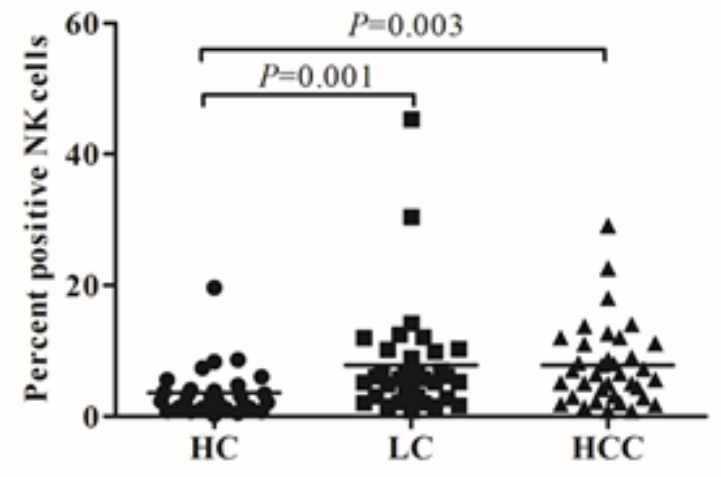

Figure 2

The proportion of activating receptors expression and inhibitors expression in NK cells. (A) The proportion of natural killer cells which expressed inhibitory receptors of CD158a/b in HC, LC and HCC subpopulation. Ns means there was no statistical significance. (B) The proportion of natural killer cells which expressed activating receptors of $\mathrm{NKp} 30 / \mathrm{p} 44 / \mathrm{G} 2 \mathrm{D} / \mathrm{p} 46$ in three groups. 
A

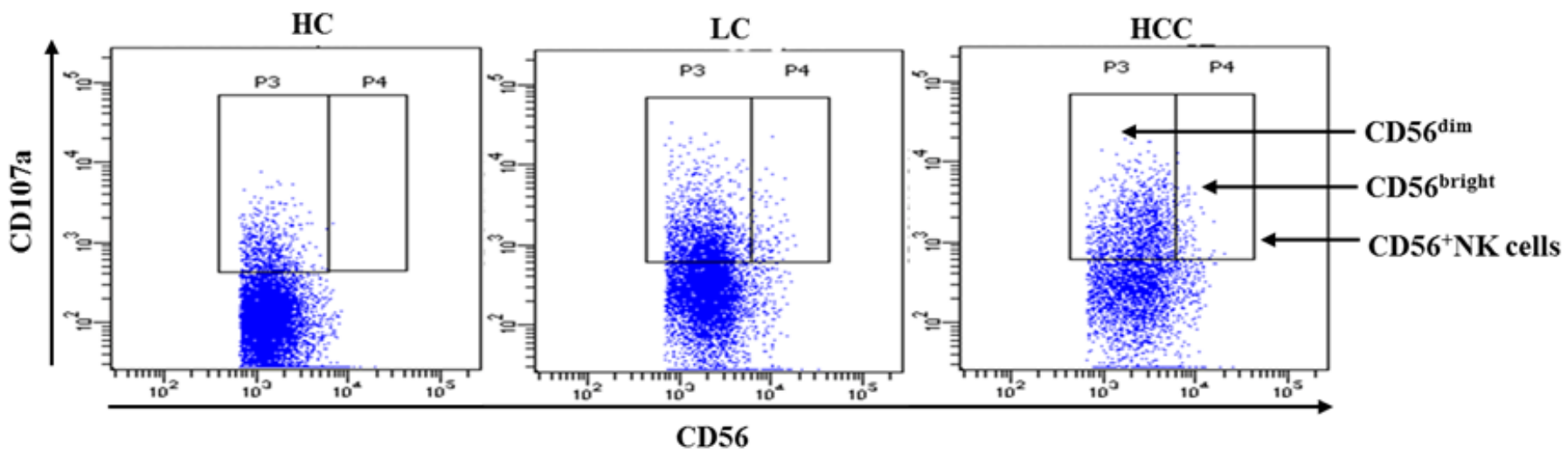

B

CD107a

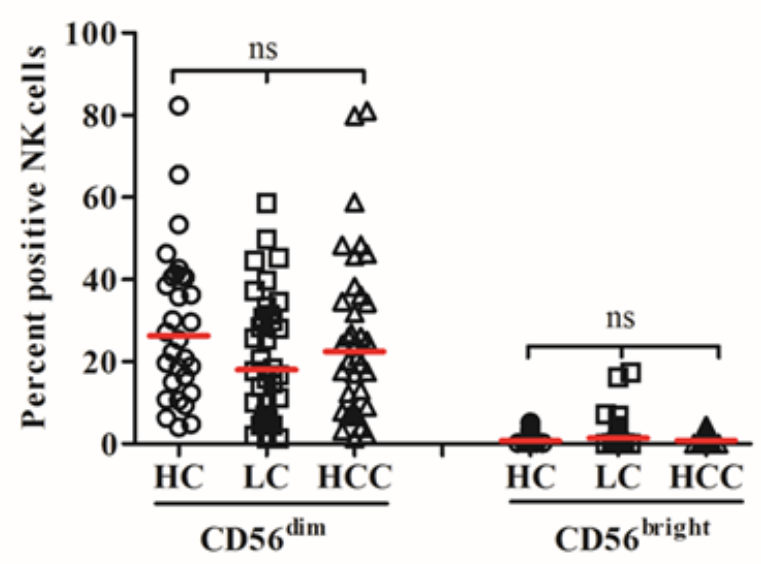

Figure 3

Degranulation of NK cells expressing CD107a. (A) Representative dot plots of NK cell expressing CD107a in three groups. (B) The percentage of NK cells expressing CD107a in three groups. Ns means there was no statistical significance. 


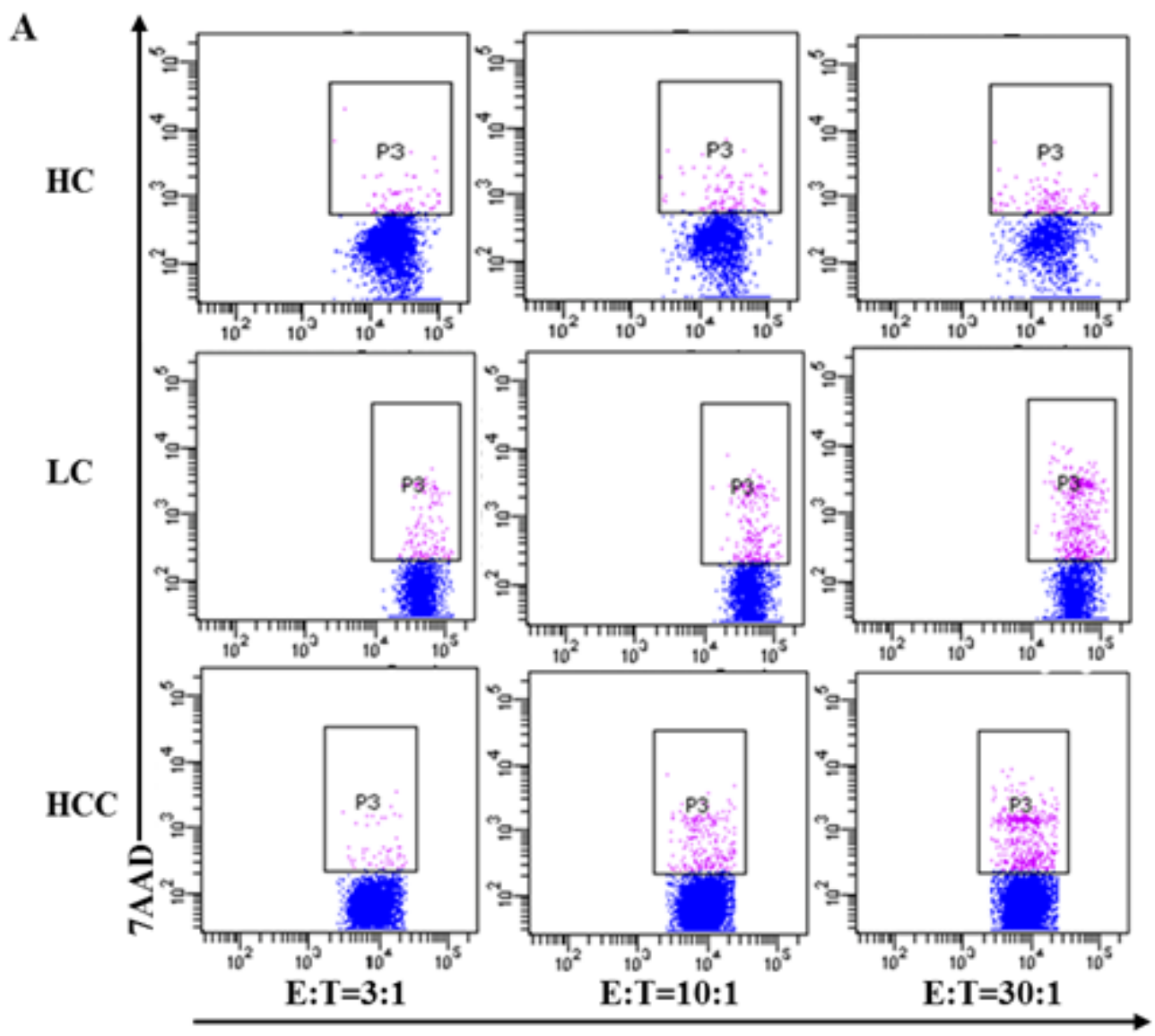

\section{CFSE}

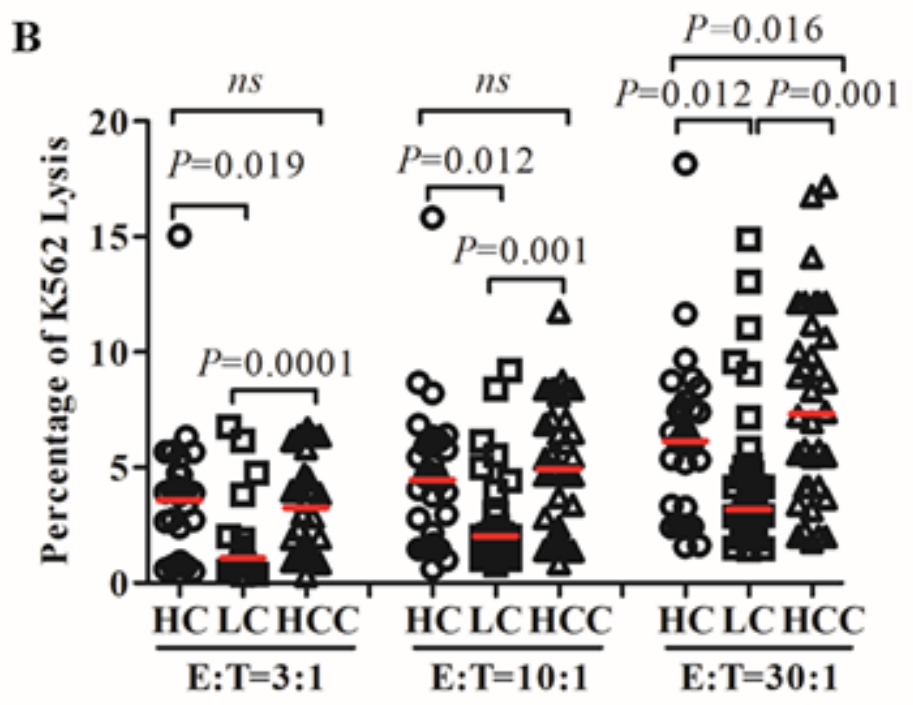

Figure 4

Cytolytic activity of NK cells among the three groups. (A) Dot plots of the K562 lysis proportion at the E:T ratio of 3:1, 10:1 and 30:1 within all the enrolled three subpopulations. (B) The proportion of K562 lysis proportion at the E:T ratio of 3:1, 10:1 and 30:1 in each subgroup, separately. Ns means there was no statistical significance. 